\title{
Daño mecánico en semillas de dos cultivares de poroto (Phaseolus vulgaris L.), impacto sobre la fisiología de la germinación
}

\author{
Mechanical harm in seeds of two cultivars of bean (Phaseolus vulgaris L.), \\ impact on the physiology of germination \\ Amalia Romano $^{1 *}$, Juan Argüello ${ }^{2}$, Irma Teves ${ }^{1}$, Nora De Pascuale ${ }^{1}$, \\ Gabriela Oddone ${ }^{3}$, Lauro Cazón ${ }^{1}$
}

\section{RESUMEN}

La calidad de la semilla decae como consecuencia del deterioro. Se analizó el impacto del daño inducido en semillas de poroto de los cultivares Perla INTA (blanco) y NAG 12 INTA (negro) sobre la fisiología de su germinación. Para ello, muestras de cada cultivar fueron deterioradas dejándolas caer sobre un plato metálico cero, dos y cuatro veces. Posteriormente, se evaluaron porcentajes de germinación, conductividad eléctrica, parámetros y tasas de crecimiento de plántulas; y la actividad de la $\alpha$-amilasa en cotiledones y embriones durante los primeros tres días de iniciada la imbibición de la semilla. Si bien en poroto blanco y negro los porcentajes de germinación no mostraron diferencias significativas respecto del testigo, solo el tratamiento con dos golpes en poroto blanco mostró incrementos significativos $(\mathrm{p} \leq 0,05)$ en la conductividad eléctrica. En cuanto al crecimiento de plántulas, en poroto negro con cuatro golpes se observaron caídas significativas en la longitud y el peso fresco del vástago. Respecto de la valoración enzimática se evidenciaron mermas en la actividad de la $\alpha$-amilasa asociadas a incrementos en los niveles de daño de la semilla, observándose que mientras en poroto blanco el deterioro promueve variaciones a nivel de cotiledones, en poroto negro el área crítica de esas modificaciones le correspondería al embrión. Los resultados indican que el daño mecánico promueve modificaciones diferenciales en la fisiología de la germinación de poroto blanco y de poroto negro y según el nivel de deterioro que presente la semilla.

Palabras clave: daño mecánico, semilla, $\alpha$-amilasa, Phaseolus vulgaris $\mathrm{L}$.

\begin{abstract}
Seed quality declines as a consequence of deterioration, the impact of harm induced to bean seeds of the cultivars Perla INTA (white) and NAG 12 INTA (black) on the physiology of their germination, has been analized. For this purpose, samples of each cultivar were deteriorated by leaving them fall an a metallic dish: none, two and four times. After wards, germination percentages, electrical conductivity, parameters and growth rate of seedlings were evaluated; as wells as the $\alpha$-amylase activity of cotiledons and embryos during the first three days after beginning of seed imbibition. Although germination percentages of white and black bean didn't show significant differences, as compared with the control, only the treatment of white bean with two blows has shown significant increases $(p \leq 0,05)$ in electrical conductivity.

Concerning the growth of black bean seedlings with four blows, significant descents in length and fresh weight of shoots have been observed. Regarding enzymatic valuation, decreases in the activity of $\alpha$-amylase became evident, associated with increases in levels of seed harm; noting that -while deterioration in black bean promotes variation in cotyledons-in black bean, critical area of these modifications would correspond to embryo.

Results indicate that mechanical harm promotes differential modifications in the germination physiology of white and black beans, and also in relation to the level of deterioration of seed.
\end{abstract}

Key words: mechanical harm, seed, $\alpha$-amylase, Phaseolus vulgaris $L$.

\section{Introducción}

Argentina aporta el $98 \%$ de la producción mundial de poroto alubia. Su cultivo en los diferentes tipos comerciales es uno de los principales rubros de la producción agrícola en las provincias de noroeste argentino (SAGPYA). El uso de semillas de alta calidad constituye uno de los principales

\footnotetext{
Universidad Nacional de Jujuy. Facultad de Ciencias Agrarias. San Salvador de Jujuy. Jujuy. Argentina.

Universidad Nacional de Córdoba. Facultad de Ciencias Agropecuarias. Córdoba. Argentina.

3 Universidad Nacional de Jujuy. Facultad de Ciencias Económicas. San Salvador de Jujuy. Jujuy.

* Autor por correspondencia: postgrado@unju.edu.ar
}

Fecha de Recepción: 10 Noviembre, 2012.

Fecha de Aceptación: 9 Septiembre, 2013. 
factores que se necesitan para alcanzar una elevada productividad a partir del establecimiento de una adecuada población de plantas (Moreano et al., 2011). En este contexto la rentabilidad del productor se ve afectada por el uso de semillas de mala calidad. Lo que acarrea en cultivos de granos pérdidas anuales superiores a los 300 millones de pesos (Casini, 2004). $\mathrm{Al}$ respecto estudios realizados en soja (Moreano et al., 2011) permiten sostener que la reducción en la calidad fisiológica de la semilla es consecuencia de problemas comunes como el envejecimiento, el deterioro provocado por la presencia de plagas y el daño mecánico. Esta última causa producida durante la cosecha y en el almacenamiento se constituyó en uno de los principales factores determinantes de las pérdidas en la calidad de semillas de algodón. Al respecto Souza et al. (2009) observaron que esas semillas con diferentes niveles de daño mecánico si bien germinaron, producían plántulas menos vigorosas y con un elevado porcentaje de anormalidades. Lo señalado sugiere que la expresión del deterioro de la semilla es variada como lo evidenciaron Romano et al. (2008) en otros cultivares de poroto blanco y negro.

Si bien las semillas presentan al momento de su madurez fisiológica, el mayor nivel de calidad, posteriormente la misma declina de manera sostenida como consecuencia del envejecimiento. Al respecto, Lewis et al. (1998) relacionan al deterioro de la semilla con una serie de cambios que suceden como las pérdidas de la integridad de membranas y de la actividad enzimática, y aumentos en daños cromosomales. Asimismo sostienen que la tasa de deterioro depende de factores ligados a la constitución genética y a las condiciones que se presentan durante la cosecha y en el almacenaje.

Investigaciones realizadas en poroto (Binotti et al., 2008) con el propósito de conocer el efecto del deterioro inducido sobre la calidad de la semilla permitieron comprobar que según aumenta el grado de daño, los valores de conductividad eléctrica también se incrementan por causa de mayores pérdidas en la integridad de las membranas, y debido a que la semilla de poroto es aleuro-amilácea se encontraron además cantidades crecientes de aminoácidos y de azúcares en el líquido de lixiviación. Lo señalado permitió corroborar que el aumento en el deterioro de la semilla al promover importantes cambios fisiológicos, afectan negativamente no solo la germinación, sino que también su vigor. Al respecto, trabajos realizados en semillas de poroto con envejecimiento artificial (Santos et al., 2004) mostraron importantes reducciones en el tamaño de las plántulas que se formaron. Por otra parte, estudios encarados en soja (Vieira et al., 2002) demostraron que cuando el contenido de humedad de la semilla era inferior al $10 \%$, los lotes mostraban mayores valores en la conductividad eléctrica, aun cuando en ellos se habían medido porcentajes similares de germinación. Estos resultados se atribuyeron a que el bajo contenido de humedad junto a una importante desorganización de las membranas, propició incrementos en la lixiviación de los solutos, lo que caracterizó el bajo vigor de la semilla.

Durante la germinación ocurren modificaciones metabólicas debido a las actividades enzimáticas que convierten a las sustancias de reserva en formas químicas fácilmente transportables. Es así como investigaciones realizadas en semillas de trigo sometidas a deterioro natural y artificial (Ganguli and Sen-Mandi, 1990) permitieron determinar que las variaciones en la germinación se deben a pérdidas en la actividad amilolítica, sosteniendo además que esas declinaciones estaban asociadas a la pérdida en la viabilidad de la semilla. Por otra parte y para la misma especie (Das and Sen-Mandi, 1992) comprobaron que durante la imbibición hubo un considerable incremento de la actividad $\alpha$-amilasa. Por otro lado, evaluaciones de esta enzima durante la germinación de semillas de Vigna (Morohashi et al., 1989) les permitió comprobar marcados incrementos en su actividad 48 horas después de iniciada la imbibición de la semilla. En tanto que los estudios realizados en Carthamus (Elarbi et al., 2009) evidenciaron que la actividad de la $\alpha$-amilasa se incrementaba hasta el $5^{\text {to }}$ día de iniciada la germinación, para posteriormente declinar de modo acelerado. Otros investigadores (Beers \& Duke, 1990) al estudiar este comportamiento en arveja verificaron que en los cotiledones la enzima aumenta su actividad por lo menos durante los primeros 10 días de germinación. Finalmente estudios realizados en soja bajo condiciones de deterioro natural y artificial (Santos et al., (2004) mostraron que el daño de la semilla promueve alteraciones en los respectivos sistemas enzimáticos.

Por otra parte, en cereales se estudió en detalles el rol que cumple el eje embrionario para inducir la síntesis de $\alpha$-amilasa en la capa de aleurona (Higgins et al., 1982). Sin embargo, en legumbres el mecanismo que se reportó para explicar el desarrollo de la actividad $\alpha$-amilasa en tejidos de reservas de la semilla, resulta contradictorio. Así mientras los 
trabajos efectuados en Vigna por Morohashi et al. (1989) mostraron que los máximos niveles de actividad de la enzima se obtuvieron en cotiledones unidos al embrión, en tanto que la actividad en cotiledones aislados esos incrementos fueron mínimos. En poroto (Dale, 1969) se comprobó que la actividad de la $\alpha$-amilasa en los cotiledones no es afectada por la separación del eje embrionario.

En otro orden de cosas, estudios comparativos realizados entre líneas de poroto blanco y coloreado con el propósito de evaluar su calidad fisiológica, determinaron que poroto blanco es más sensible al deterioro que poroto negro (Romano et al., 2002; Romano et al. 2010). Otras investigaciones abordadas para conocer sus comportamientos frente al daño mecánico permitieron comprobar mayor resistencia en las líneas de poroto de color respecto de los blancos (Dickson \& Boettger, 1976). Asimismo, otros antecedentes dan cuenta de que este tipo de deterioro merma la tasa de translocación de nutrientes hacia los tejidos de conducción y cotiledones (Pollock et al., 1969) efectos que además resultan decisivos para definir las tasas de crecimiento de raíz y de vástago.

De acuerdo con lo expuesto se propone la siguiente hipótesis: en función del tipo de resistencia que ofrece cada cultivar de poroto al deterioro, variaciones en la intensidad del daño mecánico promueven deterioro fisiológico durante la germinación, cuyos mecanismos pueden ser evaluados a través de procesos metabólicos relacionados con la movilización de polisacáridos por $\alpha$-amilasa.

El objetivo de este trabajo fue estudiar cómo el daño mecánico en semillas de poroto blanco cv. Perla INTA y de poroto negro cv. NAG 12 INTA impacta sobre la fisiología de la germinación.

\section{Materiales y Métodos}

Material biológico: Se utilizaron lotes de semillas de poroto (Phaseolus vulgaris L.) pertenecientes a un cultivar blanco Perla INTA y a otro cultivar negro NAG 12 INTA, cosecha 2009 de primera multiplicación, provistas por la estación Experimental INTA-Cerrillos (Argentina).

Tratamiento para deterioro inducido: con el propósito de disponer de semillas con diferentes niveles de daño mecánico, los lotes de cada cultivar de poroto previamente curados con captan a razón de $1 \mathrm{~g} / \mathrm{kg}$ y divididos en 3 sublotes, se dejaron caer
0,2 y 4 veces sobre un plato metálico, desde una altura de 2 metros (Dickson and Boettger, 1976).

\section{Determinaciones en laboratorio}

\section{I) Viabilidad}

Ensayo de germinación: en cada uno de 3 ensayos realizados durante el año 2010, de cada cultivar y tratamiento se sembraron 5 repeticiones de 10 semillas entre toallas de papel humedecida con agua destilada. Los rollos ubicados en bolsas de polietileno transparente se colocaron en cámara de germinación con un fotoperiodo 8:16 hrs (luz: oscuridad) a $20-30^{\circ} \mathrm{C}$. Al noveno día se evaluó el número de plantas normales, anormales y el de semillas muertas (ISTA, 2010). Los resultados se expresaron en porcentajes.

\section{II) Vigor}

Determinación de conductividad eléctrica: se colocaron a embeber entre toallas de papel húmedo semillas de poroto de cada cultivar y tratamiento por 16 horas a $4{ }^{\circ} \mathrm{C}$. A continuación, 10 ejes embrionarios de semillas de cada fracción fueron ubicados en recipientes individuales con $10 \mathrm{ml}$ de agua deionizada, agitando cada 20 minutos por espacio de 3 horas (Romano et al., 2002). Los valores de conductividad eléctrica evaluados en un Conductímetro Altronix en s. $\mathrm{cm}^{-1}$ fueron expresados en porcentajes, dividiendo el valor inicial por la conductividad eléctrica final (Stewart and Bewley, 1980).

Evaluación del crecimiento: a partir de plántulas normales obtenidas de los ensayos de germinación, se valoraron longitudes $(\mathrm{cm})$ y pesos $(\mathrm{g})$ frescos y secos de raíz y de vástago. Se calculó además la tasa de crecimiento de plántulas:

TCP $\left(\mathrm{mg}^{\mathrm{d}} \mathrm{d}^{-1}\right)=$ peso seco total $/ 9$ (Soltani et al., 2002).

\section{III) Evaluación de actividad enzimática}

Acondicionamiento de las semillas: en cajas de petri grande sobre papel humedecido con agua destilada se colocaron 5 repeticiones de 15 semillas de cada cultivar y tratamiento. Las mismas se mantuvieron en cámara de germinación con fotoperiodo 8:16 hrs (luz: oscuridad) a $20-30{ }^{\circ} \mathrm{C}$ por periodos de: $0 ; 24 ; 30 ; 48$ y 72 horas. Al término de 
cada periodo, se extrajeron las muestras respectivas para su utilización en el siguiente paso,

Preparación de los extractos: luego de separar cotiledones y eje embrionario, de cada uno se preparó un macerado acuoso en baño de agua de $37^{\circ} \mathrm{C}$ por 20 minutos, procediéndose luego a su filtrado.

A continuación en tubos de ensayos se incorporó $1 \mathrm{ml}$ de solución de almidón $500 \mathrm{mg} / \mathrm{l}$, tampón a pH 7 con buffer-fosfato $0,1 \mathrm{mg} / \mathrm{l}$ en $\mathrm{ClNa} 0,15$ $\mathrm{mol} / \mathrm{l}$. Posteriormente se incorporó $20 \mu \mathrm{l}$ de cada extracto, dejándolo en reposo en baño a $37^{\circ} \mathrm{C}$ por 7,5 minutos. Luego a cada tubo se le incorporó solución de 0,01 eq/l de iodo ácido clorhídrico 0,02 mol/l, mezclándose con suave agitación.

Al final se retiraron los tubos del baño agregándoles $8 \mathrm{ml}$ de agua destilada, para luego proceder a medir la absorbancia a $640 \mathrm{~mm}$, utilizando un espectrofotómetro marca Spectrum S 105.

Cálculo de la actividad de la $\alpha$-amilasa:

$$
\text { Amilasa }(\mathrm{UA} / \mathrm{dl})=\frac{\mathrm{C}-\mathrm{D}}{\mathrm{C}} \times 100
$$

C: absorbancia del control,

D: absorbancia de cada muestra.

Una unidad amilolítica (UA) es la cantidad de enzima contenida en $100 \mathrm{ml}$ de muestra, que puede hidrolizar $10 \mathrm{mg}$ de almidón en 30 minutos bajo las condiciones de la reacción (kit Merck).

\section{Diseño experimental}

Para cada ensayo propuesto se utilizó un diseño completamente aleatorizado con 5 repeticiones con el uso de Excel Infostat 2011. Los datos fueron analizados por ANAVA y las medias estudiadas por el test de Tukey $(\mathrm{p} \leq 0,05)$.

\section{Resultados y Discusión}

Los ensayos de germinación de poroto blanco y y poroto negro (Tabla 1) no evidenciaron diferencias significativas $(\mathrm{p} \geq 0,05)$ para los parámetros porcentajes de plántulas normales y anormales entre los diferentes niveles de deterioro que presentaban los sublotes en estudio. Esto concuerda con los resultados presentados por Romano et al. (2008) en otros cultivares de poroto blanco y negro. Si bien la germinación generalmente es un indicador sensible del nivel de daño que presenta la semilla cuando se comparan muestras con diferencias en su calidad (Lewis et al., 1998). Lo observado corrobora apreciaciones de Vieira et al. (2002) al sostener la incidencia que ejerce el contenido de humedad de la semilla sobre las valoraciones de viabilidad y vigor de la misma.

Respecto de la conductividad eléctrica, esta variable permite detectar diferencias significativas $(\mathrm{p} \leq 0,05)$ solo para el cv. Perla INTA en el nivel medio de daño ( 2 caídas) respecto de los otros niveles de deterioro ensayados. Esto confirma lo propuesto por Binotti et al. (2008), quienes sostienen que las semillas deterioradas no pueden controlar las pérdidas de solutos. Sin embargo en poroto negro no se comprobaron diferencias entre los distintos niveles de deterioro. Resultados semejantes fueron presentados por Vieira et al. (2002), quienes lo justifican al sostener que la humedad inicial de la semilla en interacción con otros factores afecta la conductividad eléctrica. Asimismo la mayor resistencia al daño observado por el cv. NAG 12 INTA, coincide con lo que sostienen Dickson and Boettger (1976), Romano et al. (2002), al corroborar que las semillas de poroto de color son más resistentes al deterioro respecto de las blancas.

Tabla 1. Calidad fisiológica de semilla de los cultivares Perla INTA y NAG 12 INTA con distintos niveles de daño mecánico.

\begin{tabular}{|c|c|c|c|c|c|c|c|c|}
\hline \multirow{3}{*}{$\begin{array}{l}\text { Niveles de daño } \\
\text { mecánico }\end{array}$} & \multicolumn{4}{|c|}{ Germinación st } & \multirow{2}{*}{\multicolumn{2}{|c|}{$\begin{array}{l}\text { Tasa de crecimiento de } \\
\text { plántulas } \\
\left(\mathrm{mg} . \mathrm{d}^{-1}\right)\end{array}$}} & \multirow{2}{*}{\multicolumn{2}{|c|}{$\begin{array}{l}\text { Conductividad eléctrica } \\
\qquad(\%)\end{array}$}} \\
\hline & \multicolumn{2}{|c|}{$\begin{array}{c}\text { Pl. normales } \\
(\%)\end{array}$} & \multicolumn{2}{|c|}{$\begin{array}{c}\text { Pl. anormales } \\
(\%)\end{array}$} & & & & \\
\hline & PI & NI & PI & NI & PI & NI & PI & NI \\
\hline Testigo & $92 \mathrm{a}$ & $72 \mathrm{a}$ & 6 & 26 & $24 \mathrm{a}$ & $11 \mathrm{a}$ & $23,91 \mathrm{~b}$ & $27,21 \mathrm{a}$ \\
\hline Medio (2 caídas) & $86 a$ & $64 \mathrm{a}$ & 14 & 34 & $23 \mathrm{a}$ & $12 \mathrm{a}$ & $28,69 \mathrm{a}$ & $26,71 \mathrm{a}$ \\
\hline Alto (4 caídas) & $82 \mathrm{a}$ & $80 \mathrm{a}$ & 18 & 20 & $24 \mathrm{a}$ & $10 \mathrm{a}$ & $25,59 \mathrm{~b}$ & $25,68 \mathrm{a}$ \\
\hline $\mathrm{CV}(\%)$ & 12,0 & 17,0 & & & 9,2 & 10,3 & 6,0 & 22,0 \\
\hline
\end{tabular}

PI: Perla INTA; NI: NAG 12 INTA.

Letras diferentes indican diferencias significativas $(p \leq 0,05)$ Tukey. 
El análisis de las plántulas normales en ambos cultivares, a nivel de raíz (Tabla 2) no registró diferencias significativas para ninguno de los niveles de daño estudiados. Sin embargo, al evaluar el vástago (Tabla 3 ) se encontraron diferencias significativas $(\mathrm{p} \leq 0,05)$ en poroto negro con el máximo nivel de daño (4 caídas) tanto en longitud como en el peso fresco del órgano. Estos resultados sostienen apreciaciones de Moreano et al. (2011) y de Souza et al. (2009) acerca de las pérdidas en la calidad de la semilla por efecto del daño mecánico, y concuerdan con lo registrado por Pollock et al. (1969) y Santos et al. (2004), quienes al estudiar el vigor a nivel de semillas y de plántulas de poroto reportan que las mermas en el crecimiento se deben a que el daño mecánico reduce la provisión de nutrientes disponibles en los cotiledones.

Por otro lado, durante los primeros tres días de iniciada la imbibición de la semilla, las determinaciones de la evolución de la síntesis de $\alpha$-amilasa tanto en ejes embrionarios como en cotiledones de poroto blanco y negro evidenciaron variaciones en el metabolismo entre los distintos niveles de daños mecánicos estudiados. Estos resultados se corresponden con lo expresado para trigo por Das and Sen-Mandi (1992) cuando sostienen que durante el periodo de imbibición de la semilla hay actividad enzimática y también para poroto por Dale (1969) al verificar que la actividad $\alpha$-amilasa del embrión y de los cotiledones es independiente.

La Figura 1 muestra que la actividad de la enzima medida en embriones de semillas de poroto blanco con 2 y 4 golpes, decae hasta las 24 horas de iniciada la imbibición, con posterior aumento hacia las 30 horas respecto de los controles. Estos resultados confirman lo encontrado en trigo por Ganguli and Sen-Mandi (1990). Estos autores postulan que la merma en la actividad $\alpha$-amilasa está asociada a incrementos en los niveles de deterioro de la semilla.

Por su parte, los cambios que se produjeron en la actividad enzimática a nivel de cotiledones se observan en la Figura 2. Si bien al iniciarse la imbibición hay actividad $\alpha$-amilasa en todos los casos, con el menor nivel de daño no se detectan variaciones en ese comportamiento respecto del control. Sin embargo, cuando el deterioro es mayor merma la actividad a las 24 horas, y alcanza luego máxima actividad a las 48 horas de iniciada la imbibición. Estos resultados evidencian que un

Tabla 2. Valores promedios de parámetros del crecimiento de raíz de plántulas normales de los cultivares Perla INTA y NAG 12 INTA con diferentes niveles de daño mecánico.

\begin{tabular}{|c|c|c|c|c|c|c|}
\hline \multirow{3}{*}{$\begin{array}{l}\text { Niveles } \\
\text { de daño mecánico }\end{array}$} & \multicolumn{6}{|c|}{ R A Í Z } \\
\hline & \multicolumn{2}{|c|}{ Longitud (cm) } & \multicolumn{2}{|c|}{ Peso fresco $(\mathrm{g})$} & \multicolumn{2}{|c|}{ Peso seco (g) } \\
\hline & PI & NI & PI & $\mathrm{NI}$ & PI & $\mathrm{NI}$ \\
\hline Testigo & $23,80 \mathrm{a}$ & $20,46 \mathrm{a}$ & $1,08 \mathrm{a}$ & $0,46 \mathrm{a}$ & $0,061 \mathrm{a}$ & $0,028 \mathrm{a}$ \\
\hline Medio ( 2 caídas) & $23,61 \mathrm{a}$ & $19,98 \mathrm{a}$ & $0,98 \mathrm{a}$ & $0,51 \mathrm{a}$ & $0,055 \mathrm{a}$ & $0,031 \mathrm{a}$ \\
\hline Alto (4 caídas) & $22,40 \mathrm{a}$ & $20,69 \mathrm{a}$ & $0,95 \mathrm{a}$ & $0,48 \mathrm{a}$ & $0,056 \mathrm{a}$ & $0,026 \mathrm{a}$ \\
\hline $\mathrm{CV}(\%)$ & 4,4 & 6,8 & 9,1 & 8,2 & 11,1 & 11,6 \\
\hline
\end{tabular}

PI: Perla INTA; NI: NAG 12 INTA.

Letras diferentes indican diferencias significativas $(\mathrm{p} \leq 0,05)$ Tukey.

Tabla 3. Valores promedios de parámetros de crecimiento de vástago de plántulas normales de los cultivares Perla INTA y NAG 12 INTA con diferentes niveles de daño mecánico.

\begin{tabular}{lcccccc}
\hline \multirow{2}{*}{$\begin{array}{l}\text { Niveles } \\
\text { de daño mecánico }\end{array}$} & \multicolumn{5}{c}{ Longitud $(\mathrm{cm})$} & \multicolumn{2}{c}{ Peso fresco $(\mathrm{g})$} & \multicolumn{2}{c}{ Peso seco $(\mathrm{g})$} \\
\cline { 2 - 7 } & PI & NI & PI & NI & PI & NI \\
\cline { 2 - 7 } & $23,07 \mathrm{a}$ & $27,26 \mathrm{a}$ & $2,86 \mathrm{a}$ & $1,35 \mathrm{a}$ & $0,167 \mathrm{a}$ & $0,080 \mathrm{a}$ \\
Testigo & $23,88 \mathrm{a}$ & $28,13 \mathrm{a}$ & $2,73 \mathrm{a}$ & $1,47 \mathrm{a}$ & $0,155 \mathrm{a}$ & $0,081 \mathrm{a}$ \\
Medio (2 caídas) & $20,43 \mathrm{a}$ & $25,00 \mathrm{~b}$ & $2,71 \mathrm{a}$ & $1,26 \mathrm{~b}$ & $0,164 \mathrm{a}$ & $0,069 \mathrm{a}$ \\
Alto (4 caídas) & 9,8 & 5,9 & 8,4 & 8,2 & 9,5 & 14,3 \\
CV (\%) & 9,8 & & & & & \\
\hline
\end{tabular}

PI: Perla INTA; NI: NAG 12 INTA.

Letras diferentes indican diferencias significativas $(p \leq 0,05)$ Tukey. 


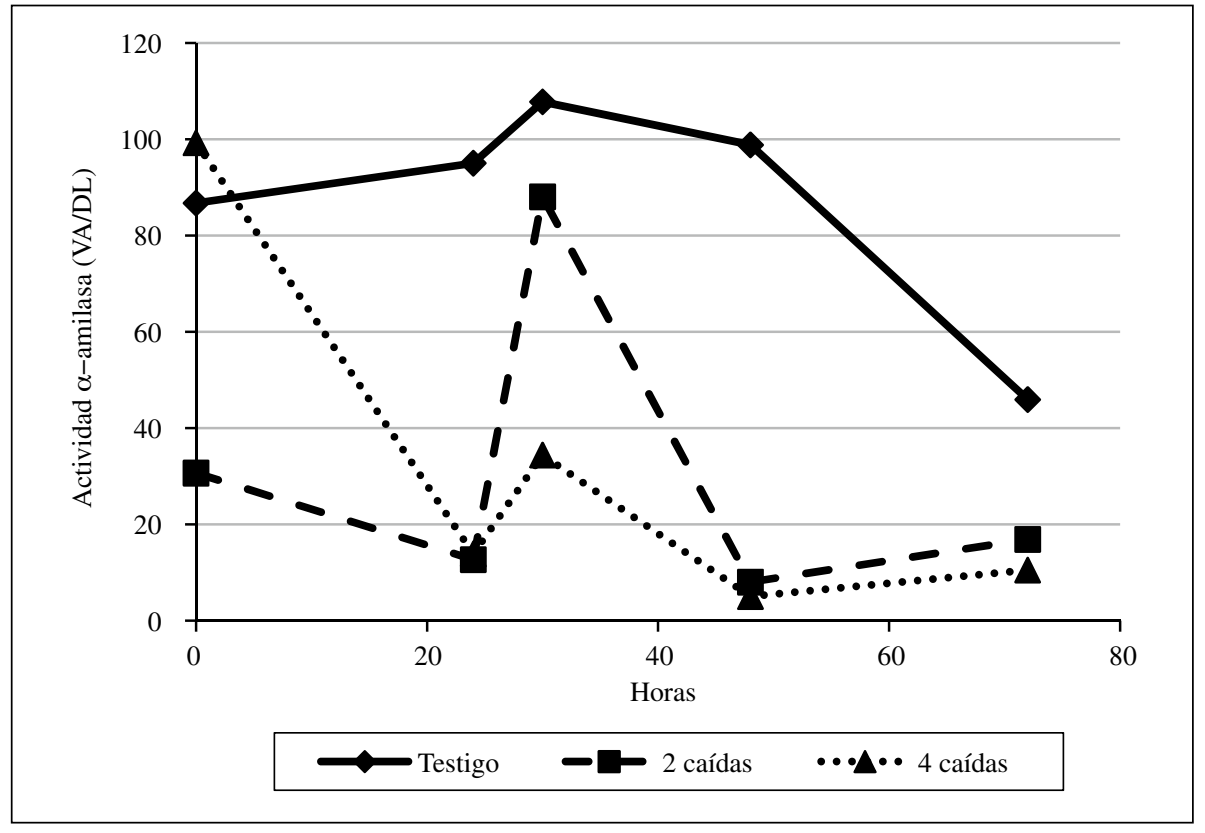

Figura 1. Actividad $\alpha$-amilasa en ejes embrionarios de poroto blanco cv Perla INTA.

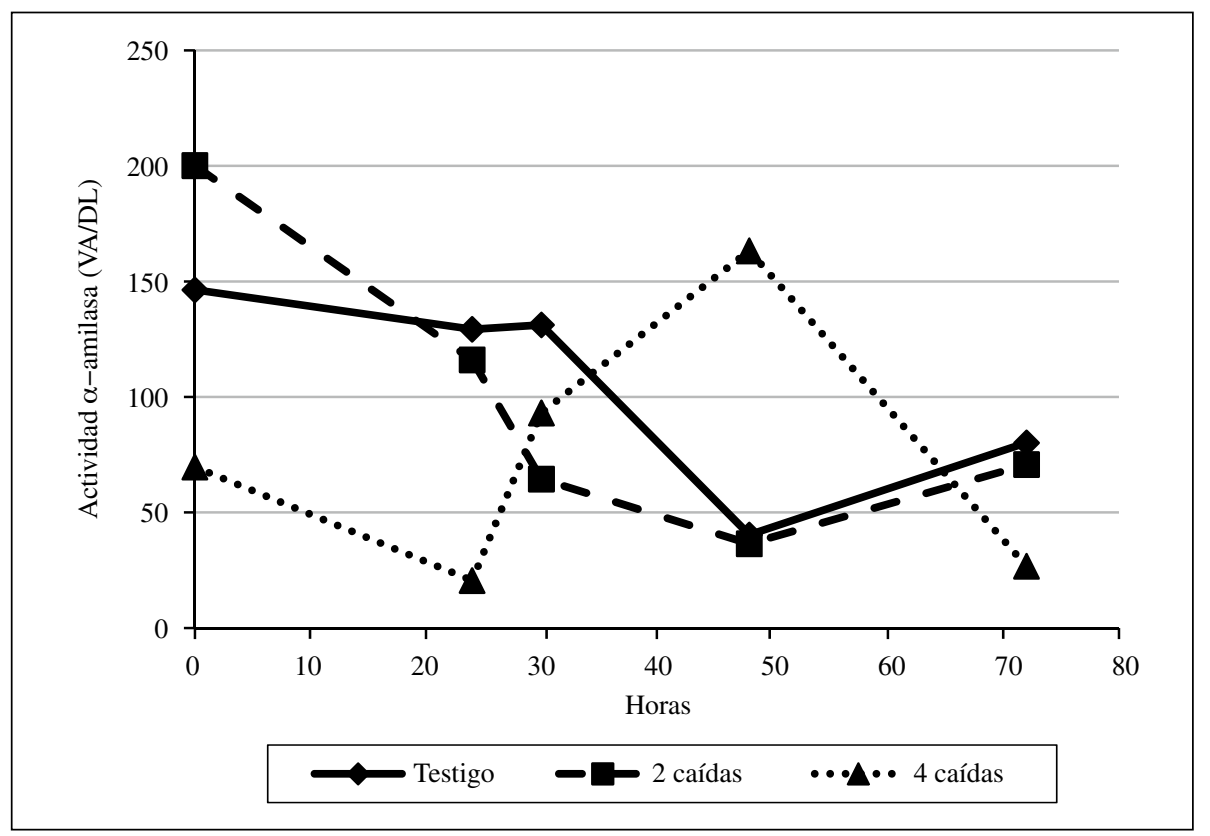

Figura 2. Actividad $\alpha$-amilasa en cotiledones de poroto blanco cv Perla INTA. 
mayor deterioro de la semilla promueve alteraciones en la actividad de esta enzima. Ello concuerda con reducciones en actividades enzimáticas encontradas en poroto por Santos et al. (2004).

En semillas de poroto negro con distintos grados de deterioro, la actividad $\alpha$-amilasa a nivel de ejes embrionarios y de cotiledones se observan en las Figuras 3 y 4 respectivamente. En el embrión esa actividad para 2 golpes y el control decae a las 24 horas, mientras que en el mayor nivel de deterioro se observa un importante incremento de la $\alpha$-amilasa. Posteriormente todos los tratamientos mostraron aumentos hacia el $2^{\text {do }}$ día de iniciada la imbibición. Por su parte los cotiledones del testigo y con 4 golpes evidenciaron incrementos a las 24 horas, en tanto que con 2 caídas ese aumento se expresó a las 30 horas de iniciada la imbibición. Estos resultados son semejantes a los encontrados en trigo por Ganguli and Sen-Mandi (1990) quienes sostienen que el daño de la semilla propicia variaciones en la actividad enzimática, lo que además limita la disponibilidad de sustratos requeridos para la germinación.

Si bien no se conocen antecedentes del comportamiento evidenciado por la actividad $\alpha$-amilasa durante la germinación de semillas de poroto con daño mecánico, los resultados presentados indican que hay actividad de esta enzima desde el inicio de la imbibición de la semilla. Estas observaciones se corresponden con apreciaciones realizadas por Beer and Duke (1990) en Pisum sativum L. y por Elarbi et al. (2009) en Carthamus tinctorius $\mathrm{L}$.

Los resultados aquí presentados permiten concluir que si bien el deterioro promueve alteraciones en la viabilidad y el vigor de la semilla, la sensibilidad de ésta depende de su contenido de humedad. Por otra parte las evaluaciones sobre la actividad $\alpha$-amilasa permiten reconocer que el daño mecánico mientras en poroto blanco promueve importantes variaciones en esa actividad en cotiledones, en poroto negro al parecer el área crítica de esas modificaciones le corresponde al eje embrionario.

Por lo expuesto y en las condiciones en las que se desarrolló este trabajo se postula que el daño mecánico promueve modificaciones diferenciales en la fisiología de la germinación entre semillas de poroto blanco cv. Perla INTA y de poroto negro cv. NAG 12 INTA según el grado de deterioro que presenten.

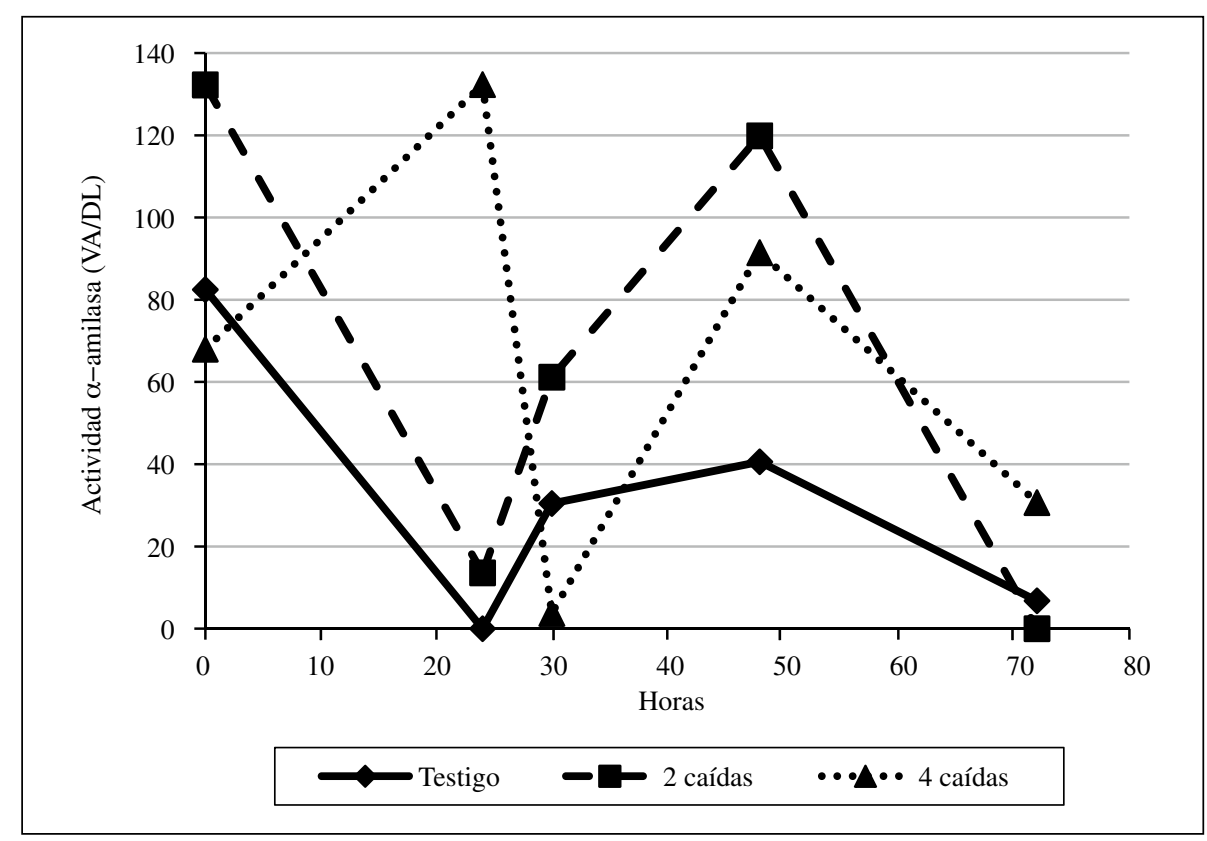

Figura 3. Actividad $\alpha$-amilasa en ejes embrionarios de poroto negro cv NAG INTA. 


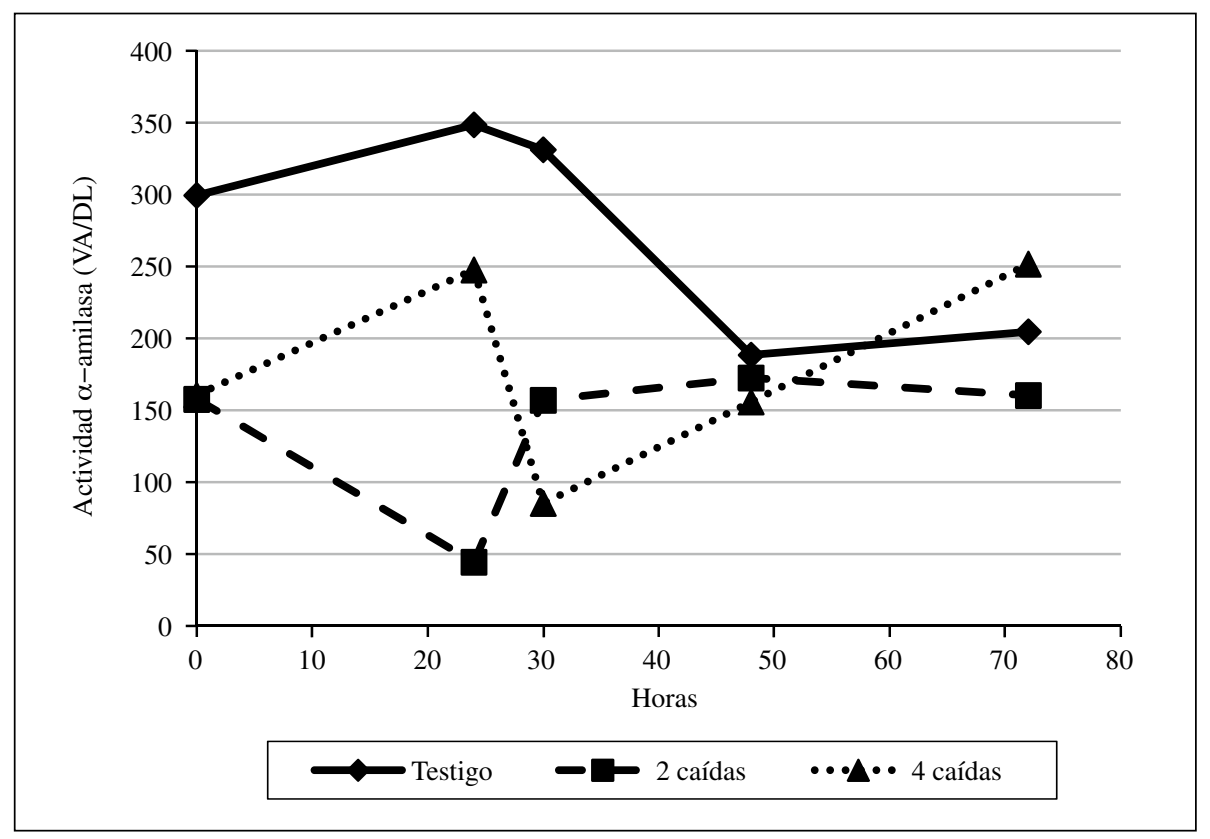

Figura 4. Actividad $\alpha$-amilasa en cotiledones de poroto negro cv NAG INTA.

\section{Literatura Citada}

Beers, E.; Duke, S.

1990. Characterization of $\alpha$ - amylase from shoots and cotyledons of pea (Pisum sativum L.) seedlings. Plant Physiol. 92: 1154-1163.

Binotti, F.; Haga, K.; Cardoso, E.; Alves, Ch.; M. de Sa; Arf O. 2008. Efeito do periodo de envelhecimiento acelerado no teste de conductividade elétrica e na qualidade fisiológica de sementes de feijao. Acta Sci Agron. Maringa, 30 (2): 247-254.

Casini, C.

2004. Una buena cosecha comienza con una Buena semilla. Boletín de Divulgación. INTA. EEA. Manfredi. 32-33.

Dale, J.

1969. Gibberellins and early growth in seedlings of Phaseolus vulgaris L. Planta 89: 155-158.

Das, G.; Sen-Mandi, S.

1992.Scutellar amylase activity in naturally and artificially aged wheat seeds. Annals of Botany 69: 497-501.

Dickson, M.; Boettger, M.

1976. Factors associated with resistance mechanical damage in snap beans (Phaseolus vulgaris L.). J. Amer. Soc. Hort. Sci, 101 (5): 541-544.

Elarbi, M.; Khemiri, H.; Jridi, T.; Hamida, J.

2009. Purification and characterization of $\alpha$-amylase from safflower (Carthamus tinctorius L.) germinating seeds. C.R. Biologies, 332: 426-432.

Ganguli, S.; S. Sen-Mandi

1990. Some physiological differences between naturally and artificially aged wheat seeds. Seed Sci and Technol. 18: 507-514.
Higgins, T.; Jacobsen, J.; Zwar, J.

1982. Gibberellic acid and abscisic acid modulate protein synthesis and mRNA levels in barley aleurone layers. Plant Mol Biol. 1: 191-215.

International Rules for Seed Testing

2010. Published by the International Seed Testing Association. Zürichstr.50.CH-8303. Bassersdorf, Switzerland.

Lewis, D.; Marshall, A.; Hides, D.

1998. Influence of storage conditions on seed germination and vigour of temperature forage species. Seed Sci \& Tech. 26: 643.655 .

Moreano, T.; Braccini, A.; Scapim, C.; Krzyzanowski, F.; Franca-Neto, J.; Masques, O.

2011. Changes in the effects of weatering and mechanical damage on soybean seed during storage. Seed Sci \& Tech. 39: 604-611.

Morohashi, Y.; Katoh, H.; Kaneko, Y.; Matsushima, H.

1989. Control of $\alpha$-amylase development in cotyledons during and following germination of mung bean seeds. Plant Physiol. 91: 253-258.

Pollock, B.; Roos, E.; Manalo, J.

1969. Vigor of garden bean seeds and seedling influenced by initial seed moisture, substrate oxygen and imbibitions temperature. J. Amer. Soc. Hort. Sci. 94 (6): 577-584.

Romano, A.S.; Argüello, J.A.; Pérez, M.A.; Teves, I.

2002. Influencia del envejecimiento acelerado en semillas de poroto (Phaseolus vulgaris L.) de los cultivares Perla INTA y Camba INTA. Información Tecnológica. 13 (1): 91-95. 
Romano, A.; Teves, I.; Torres, N.; Cazón, L.

2008. Variaciones en la calidad de semillas de poroto (Phaseolus vulgaris L.) por efectos del daño mecánico y su influencia en el vigor de las plántulas. Idesia, 26 (2): 83-87.

Romano, A.; Teves, I.; Cazón, L.

2010. Incidencia del daño mecánico en semillas de poroto (Phaseolus vulgaris L.) sobre el desarrollo y el rendimiento de plantas normales y anormales. Idesia, 28(2): 67-74.

SAGPYA

Estimaciones agrícolas. Poroto. http: //sagpya.mecon.gov.ar Consultado: 30/08/2010.

Santos, C.M.R.; Menezes, N.L.; Villela, F.A.

2004. Alteraçoes fisiológicas e bioquímicas em sementes de feijao envelhecidas artificialmente. Revista Brasileira de Sementes, 26 (1): 110-119.
Stewart, S.; Bewley, L.

1980. Lipid peroxidation associated with accelerated aging of soybean axes. Plant Physiol, 65: 245-248.

Soltani, A.; Galeshi, S.; Zeinali, E.; Latifi, N.

2002. Germination, seed reserve utilization and seedling growth of chipea as affected by salinity and seed size. Seed Sci. \& Technol. 30: 51-60.

Souza, D.; Albuquerque, M.; Zorato, M.; Carvahlo, D.

2009. Análise de daños mecánicos e qualidade de sementes de algodoeiro. Revista Brasileira de Sementes, 31 (3): 123-131.

Vieira, R.; Penariol, A.; Perecin, D.; Panobianco, M.

2002. Condutividade elétrica e teor de agua inicial das sementes de soja. Pesquisa Agropecuária Brasileira, 37: 1333-1338. 
